\title{
Photochemical Oxidation of Oil Reduced the Effectiveness of Aerial Dispersants Applied in Response to the Deepwater Horizon Spill
}

\author{
Collin P. Ward, ${ }^{* \dagger}$ Cassia J. Armstrong, ${ }^{\dagger,}$ Robyn N. Conmy, ${ }^{\S}$ Deborah P. French-McCay, \\ and Christopher M. Reddy
}
${ }^{\dagger}$ Department of Marine Chemistry and Geochemistry, Woods Hole Oceanographic Institution, Woods Hole, Massachusetts 02543, United States
${ }^{\ddagger}$ Department of Chemistry, Trinity College, Hartford, Connecticut 06106, United States
${ }^{\S}$ National Risk Management Research Laboratory, Office of Research and Development, U.S. Environmental Protection Agency, Cincinnati, Ohio 45268, United States
"RPS ASA, South Kingstown, Rhode Island 02879, United States

\section{Supporting Information}

\begin{abstract}
Chemical dispersants are one of many tools used to mitigate the overall environmental impact of oil spills. In principle, dispersants break up floating oil into small droplets that disperse into the water column where they are subject to multiple fate and transport processes. The effectiveness of dispersants typically decreases as oil weathers in the environment. This decrease in effectiveness is often attributed to evaporation and emulsification, with the contribution of photochemical weathering assumed to be negligible. Here, we aim to test this assumption using Macondo well oil released during the Deepwater Horizon spill as a case study. Our results indicate that the effects of photochemical weathering

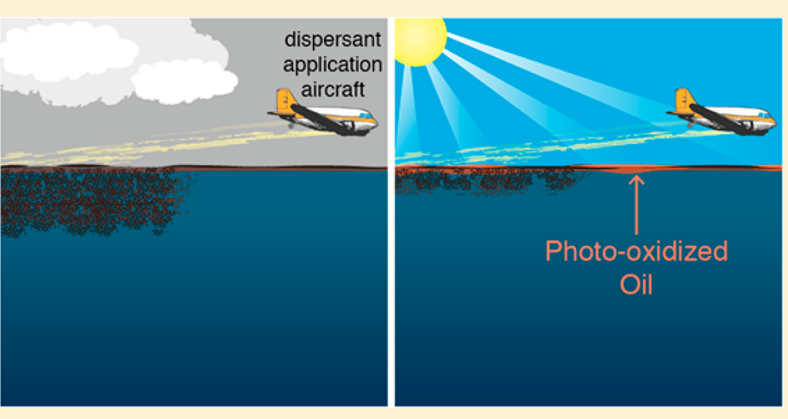
on Deepwater Horizon oil properties and dispersant effectiveness can greatly outweigh the effects of evaporative weathering. The decrease in dispersant effectiveness after light exposure was principally driven by the decreased solubility of photo-oxidized crude oil residues in the solvent system that comprises COREXIT EC9500A. Kinetic modeling combined with geospatial analysis demonstrated that a considerable fraction of aerial applications targeting Deepwater Horizon surface oil had low dispersant effectiveness. Collectively, the results of this study challenge the paradigm that photochemical weathering has a negligible impact on the effectiveness of oil spill response and provide critical insights into the "window of opportunity" to apply chemical dispersants in response to oil spills in sunlit waters.
\end{abstract}

\section{INTRODUCTION}

An effective oil spill response reduces risks to humans and ecosystems. ${ }^{1,2}$ Aerial application of chemical dispersants to floating oil during and after spills is one tool used to minimize such risks. In principle, chemical dispersants are designed to reduce the oil-water interfacial tension allowing oil to form small oil droplets that disperse into the water column. Dispersed oil is subject to multiple fate and transport processes, including microbial degradation, dissolution, volatilization, and sedimentation, thereby decreasing the amount of oil that reaches sensitive coastal shorelines. ${ }^{1,2}$ While the effectiveness of dispersants applied to the water surface can be measured quantitatively in controlled laboratory conditions, ${ }^{3}$ quantitative evidence for high dispersant effectiveness of surface applications under field conditions is limited. For example, 56\% of dispersants used in response to the 2010 Deepwater Horizon (DWH) spill in the northern Gulf of Mexico were applied to surface oil. ${ }^{4}$ In total, 412 sorties applied 972,880 gal of dispersants to surface oil over 61 days (April 22 to July 19, 2010). ${ }^{4}$ Two independent assessments of the effectiveness of aerial dispersant applications, based on aerial reconnaissance and water column fluorescence intensity (i.e., Ocean Imaging and SMART, respectively), proved inconclusive. ${ }^{5}$ Consequently, the "response" community was unable to reach consensus regarding the effectiveness of aerial dispersants applied in response to the DWH spill. ${ }^{5}$

The effectiveness of dispersants is a function of the physical (e.g., viscosity) ${ }^{6}$ and chemical (e.g., relative resin and asphaltene content $)^{7}$ properties of oil, which change when oil is subjected to weathering processes. Often, the influence of weathering on these properties has been attributed to evaporation and emulsification, with photochemical oxidation assumed to play a minor role. ${ }^{1,2,8-10}$ Photochemical weathering is not often cited as a critical weathering process when considering dispersant use, as indicated by oil spill response

Received: February 16, 2018

Revised: $\quad$ March 17, 2018

Accepted: March 22, 2018

Published: April 25, 2018 
guides $^{8}$ that support Federal On-Scene Coordinators during marine spills. Furthermore, the 2014 ExxonMobil oil spill response field manual states that the impact of photochemical weathering is "negligible relative to other weathering mechanisms", such as evaporation, a weathering process that "affects the selection of response options". ${ }^{10}$ Using the DWH spill as a case study, we showed that photochemical oxidation was a dominant weathering process that oxidized more than half of the floating oil within hours to days. ${ }^{11}$ Despite the rapid and extensive changes to DWH surface oil properties caused by sunlight, ${ }^{11}$ our understanding of how these changes impact aerial dispersant effectiveness is limited. In this study, we aim to address this gap in knowledge by (i) assessing the effect of photochemical weathering on dispersant effectiveness, (ii) comparing the effect of photochemical to evaporative weathering on dispersant effectiveness, and (iii) characterizing the effect of photochemical weathering on the effectiveness of aerial dispersants applied in response to the DWH oil spill.

\section{MATERIALS AND METHODS}

Photochemical Incubations. Macondo well oil (Source Oil B; chain of custody transfer on September 21, 2016, from $\mathrm{BP}$ to Woods Hole Oceanographic Institution) was artificially weathered in the dark at $65{ }^{\circ} \mathrm{C}$ to achieve $8,15,20$, and $30 \%$ evaporative mass losses, representative of carbon numbers 7,8 , 9, and 12, respectively. ${ }^{12}$ Aliquots of each artificially weathered DWH oil were pipetted into $150 \mathrm{~mm}$ wide precombusted Pyrex petri dishes. The dishes were leveled to maintain a uniform optical path length throughout the incubation and placed in a solar simulator for up to $24 \mathrm{~h}$ alongside dark controls (Atlas Suntest XLS+). Two steps were taken to minimize additional evaporation during the incubation. First, the dishes rested on top of a custom-made circulating water bath set at $15{ }^{\circ} \mathrm{C}$. Second, a custom-made borosilicate glass pane $[\sim 85 \%$ transmission at $320 \mathrm{~nm}$ (Figure S1)] was placed directly above the dishes to minimize the amount of infrared light the oil absorbed and to provide a barrier from the cooling fan in the experimental chamber. The temperature of the oil was monitored throughout the incubation and averaged $21 \pm 1$ ${ }^{\circ} \mathrm{C}(N=10, \pm$ standard deviation $)$. Following the incubations, the dark-control and light-exposed oil was recovered for chemical, physical, and dispersant effectiveness analyses. A detailed description of calculations comparing the rate of simulated sunlight absorption by oil in the laboratory to natural sunlight absorption by oil floating on the Gulf of Mexico in the summer of 2010 is described in Appendix S1.

Characterizing Oil Properties. The physical (i.e., viscosity, density, and adhesion) and chemical [i.e., oxygen content determined by Fourier transform infrared spectroscopy (FTIR)] properties of the dark-control and light-exposed oil were quantified. A temperature-controlled microVISC viscometer (RheoSense, Inc.) was used to determine the viscosity of the dark-control and light-exposed oil at $25^{\circ} \mathrm{C}$. Density was measured at room temperature $\left(22{ }^{\circ} \mathrm{C}\right)$ using a gastight Hamilton $250 \mu \mathrm{L}$ syringe and a NIST-calibrated analytical balance (Mettler Toledo). Aliquots of oil $(50 \mu \mathrm{L})$ were drawn into the syringe, and the volume was weighed by injecting the oil into a vial resting on the balance. Adhesion of oil (milligrams of oil per square millimeter) was determined following an iteration of a previously described method. ${ }^{13}$ Preweighed solvent-cleaned, stainless steel rods $(1.59 \mathrm{~mm}$ diameter; K\&S Precision Metals) were submerged in gas chromatography vial inserts containing $200 \mu \mathrm{L}$ of oil. After being submerged for $30 \mathrm{~s}$, the rods were placed in a draft shield for $30 \mathrm{~min}$ and then reweighed. Adhesion was calculated as the milligrams of oil adhered to the rod divided by the surface area of the portion of the rod that was submerged in the oil ( 8 $\mathrm{mm}^{2}$ ). FTIR stretching of the oil was determined as described previously. ${ }^{11,14,15}$ Briefly, the dark-control and light-exposed oil was dissolved in spectrophotometric grade trichloroethylene, transferred to a potassium bromide liquid infrared cell $(390 \mu \mathrm{m}$ path length; Harrick Scientific), and analyzed using a Bruker Vertex 70 spectrophotometer. Carbonyl stretching (intensity at $1712 \mathrm{~cm}^{-1}$ normalized to intensity at $2925 \mathrm{~cm}^{-1}$ ) was quantified because the stretching of this functional group is linearly correlated to the bulk oxygen content of DWH surface oil $\left(R^{2}=0.98 ; N=34\right){ }^{11}$

Quantifying Dispersant Effectiveness. Dispersant effectiveness (DE) was measured at room temperature using the baffled flask test (BFT), a method recommended by the U.S. Environmental Protection Agency. ${ }^{6,16}$ As previously described, ${ }^{6,16}$ the BFT uses optical spectroscopy to quantify the percentage of an oil film that is dispersed in the water column following the application of chemical dispersants and turbulent mixing. The BFT is cited within the Federal Register notice (80 FR 3380) for the proposed amendments to the 40 CFR $\S$ 300.900-920 Subpart J of the National Oil and Hazardous Substances Pollution Contingency Plan (NCP) and was recently adopted by the United Kingdom for benchtop assessments of dispersant effectiveness. ${ }^{17}$ For light sweet crude oils, the BFT yielded results similar to those of the benchtop test developed by ExxonMobil (EXDET) and largescale tests at the Bureau of Safety and Environmental Enforcement Ohmsett wave tank facility. ${ }^{18}$ COREXIT EC9500A was used in all dispersant treatments and applied at a 1:20 dispersant:oil ratio, consistent with the manufacturer's recommendation for surface application. A detailed description of the methods for characterizing the effect of sunlight on the dispersant effectiveness of DWH floating oil is described in Appendix S2.

\section{RESULTS AND DISCUSSION}

Effects of Sunlight Exposure and Evaporation on Oil Properties. Photochemical changes to the physical and chemical properties of DWH oil were examined by exposing it to increasing durations of simulated sunlight. The oxygen content of the oil increased with increasing light exposure, as determined by the intensity of infrared stretching of carbonyl functional groups. Carbonyl stretching increased from $<1 \%$ in the initial oil to $\sim 3 \%$ in oil exposed to simulated sunlight for 24 $\mathrm{h}$ [equivalent to natural sunlight for $53 \mathrm{~h}$ (Figure 1 and Figure S2)], corresponding to a 3 -fold increase in bulk oxygen content. ${ }^{11}$ The viscosity, density, and adhesion of oil increased with light exposure (Table S1). Viscosity increased nearly 7 fold after light exposure, from $22 \pm 1$ to $147 \pm 2 \mathrm{cP}$ [Table S1; \pm standard error (SE); $N=4]$. Density increased from $0.88 \pm$ $<0.01$ to $0.92 \pm<0.01 \mathrm{~g} / \mathrm{mL}$ after sunlight exposure (Table S1; \pm SE; $N=4$ ). Adhesion increased 6-fold from $0.09 \pm 0.01$ to $0.55 \pm 0.05 \mathrm{mg} \mathrm{mm}^{-2}$ (Table S1; \pm SE; $N=3$ ).

Compared to photo-oxidation, changes in oil properties due to evaporative weathering were minimal (Figure 2 and Table S2). Evaporation in the dark to $30 \%$ mass loss did not result in a change in carbonyl stretching intensity, while sunlight exposure increased carbonyl stretching by $1.8-2 \%$ (Table S2). The preferential evaporation of small, light-end hydrocarbons increased the viscosity from 9 to $39 \mathrm{cP}$; however, this 


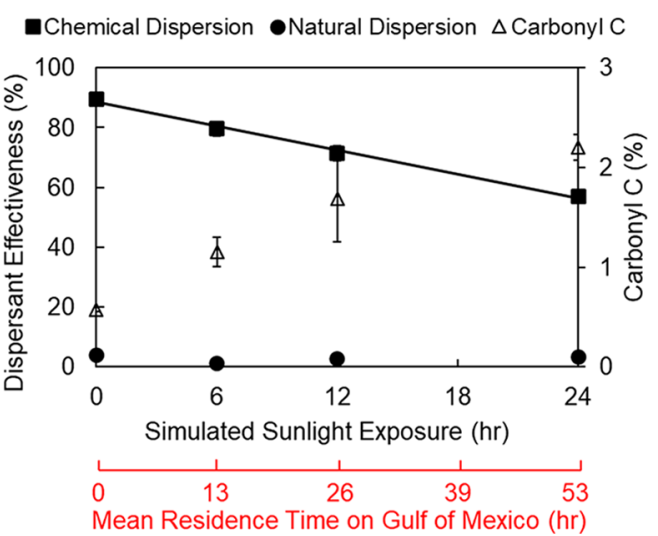

Figure 1. Changes in the chemical dispersion $(\boldsymbol{\square})$, natural dispersion $(\mathbf{O})$, and carbonyl content of oil $(\triangle)$ exposed to increasing durations of simulated sunlight. Chemical dispersion represents the fraction of oil that dispersed upon addition of COREXIT EC9500A to the photooxidized oil at a dispersant:oil ratio of 1:20. Natural dispersion treatments represent the fraction of oil that dispersed without addition of COREXIT EC9500A, which was always $<5 \%$. Carbonyl content represents infrared stretching at $1712 \mathrm{~cm}^{-1}$ normalized to $-\mathrm{CH}_{2}$ stretching at $2925 \mathrm{~cm}^{-1}$. The standard error for both dispersion treatments is sometimes smaller than the symbol $(N=4$ for chemical dispersion, and $N=2$ for natural dispersion). Error bars for carbonyl content represent the standard error of duplicate measurements. A comparison of the rate of light absorption of simulated sunlight in the laboratory experiments vs natural sunlight on the Gulf of Mexico is presented in Figure S2.

increase was much smaller than the 10 -fold increase that resulted from photochemical oxidation (Table S2). Evaporation and sunlight exposure increased density, but the increase was consistently larger for sunlight exposure (Table S2). Collectively, these results demonstrate that changes to the physical and chemical properties by short-term doses of sunlight greatly outweigh changes in bulk properties by upward of $30 \%$ evaporation in the dark.

Effects of Sunlight Exposure and Evaporation on Dispersant Effectiveness. The decrease in DE due to photooxidation was at least 4-fold larger than the decrease due to evaporation. The initial oil had $93 \pm 1 \%$ DE [Figure 1; $\pm 95 \%$ confidence interval (CI); $N=4]$, similar to previous reports for South Louisiana crude oil of $94 \pm 8 \%$ DE $( \pm 95 \%$ CI; $N=4){ }^{6}$ Dispersant effectiveness decreased linearly with increasing light exposure (Figure 1). Simulated sunlight exposure for $24 \mathrm{~h}$, which equated to natural sunlight for $53 \mathrm{~h}$ on the Gulf of Mexico in the summer (Figure 1 and Figure S2), decreased DE by $29-34 \%$. In contrast, evaporation of $8-30 \%$ of the initial mass of oil resulted in a reduction in DE of $3-7 \%$ (Figure 2). Comparatively, another study indicated $20 \%$ evaporation of Alaskan North Slope crude resulted in no significant change in DE. ${ }^{6}$ Collectively, photochemical oxidation decreased the DE of DWH oil on time scales of hours to days, and the impact of sunlight on DE was significantly greater than that of evaporation ( $p=4 \times 10^{-6}$; two-tailed, paired $t$ test).

Comparison of Laboratory Predictions versus Field Observations. The effects of sunlight on DE in the laboratory were comparable to those observed in the field. If we account for irradiance variability (Figure S2) and assume DE decreases linearly with increasing light exposure (Figure 1), our laboratory results predict that the $\mathrm{DE}$ of $\mathrm{DWH}$ surface oil should be negligible after the oil has floated on the sea surface for 5-8 days. In good agreement with the laboratory
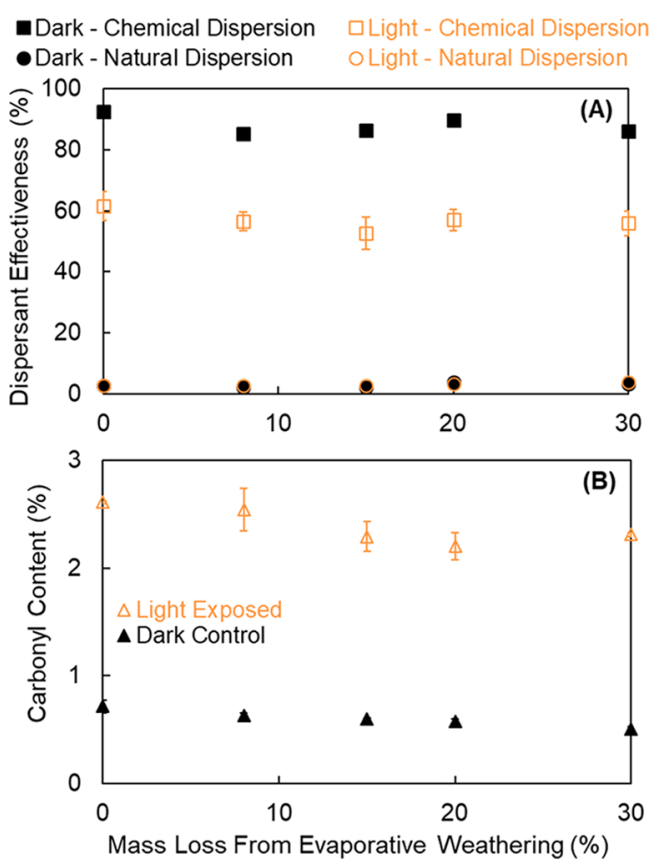

Figure 2. (A) Comparing the effects of photochemical oxidation and evaporation on dispersant effectiveness. The $x$-axis represents the initial degree of laboratory weathering due to evaporation in the dark, which ranged from 0 to $30 \%$ mass loss. The black and orange squares represent chemical dispersion of the dark-control and light-exposed oils, respectively. The black and orange circles represent natural dispersion of the dark-control and light-exposed oils, respectively. Natural dispersion treatments represent the fraction of oil that dispersed without addition of COREXIT EC9500A, which was always $<5 \%$. The error bars for all dispersion treatments represent the standard error from the mean ( $N=4$ for chemical dispersion, and $N=$ 2 for natural dispersion) and are sometimes smaller than the symbol. (B) Comparing the effects of photochemical oxidation and evaporation on carbonyl $\mathrm{C}$ content. The black and orange triangles represent carbonyl $\mathrm{C}$ content of the dark-control and light-exposed oils, respectively. Error bars for carbonyl content represent the standard error of duplicate measurements.

predictions, the $\mathrm{DE}$ of a photo-oxidized surface oil residue that floated on the Gulf of Mexico for 7-8 days before being collected was $2 \pm 1 \%\left( \pm \mathrm{SD} ; N=4\right.$; Slick B "Juniper"). ${ }^{11}$ While additional data are needed to test the assumption that $\mathrm{DE}$ decreases linearly with increasing residence time on the sunlit sea surface, the similarity of the laboratory predictions and field observations suggests this assumption is a minor source of uncertainty. The overlap between laboratory predictions and field observations indicates that sunlight was likely a primary driver in decreasing the effectiveness of aerial dispersants applied in response to the DWH oil spill.

What Is Driving the Low Dispersant Effectiveness of Photo-Oxidized Oil? Several lines of evidence suggest that the decrease in $\mathrm{DE}$ after sunlight exposure was driven by photochemical changes to the chemical rather than physical properties of oil. First, on the basis of the inverse relationship between oil viscosity and $\mathrm{DE},{ }^{6}$ the $\sim 100 \mathrm{cP}$ increase in viscosity after sunlight exposure (Table S2) would have a negligible effect on DE. Second, sunlight exposure alters the chemical behavior of DWH oil. ${ }^{11,15,19,20}$ Nonweathered DWH oil is primarily comprised of compounds that are soluble in hexane and amenable to gas chromatographic analysis. ${ }^{12}$ In contrast, photochemically oxidized oil residues are primarily comprised 


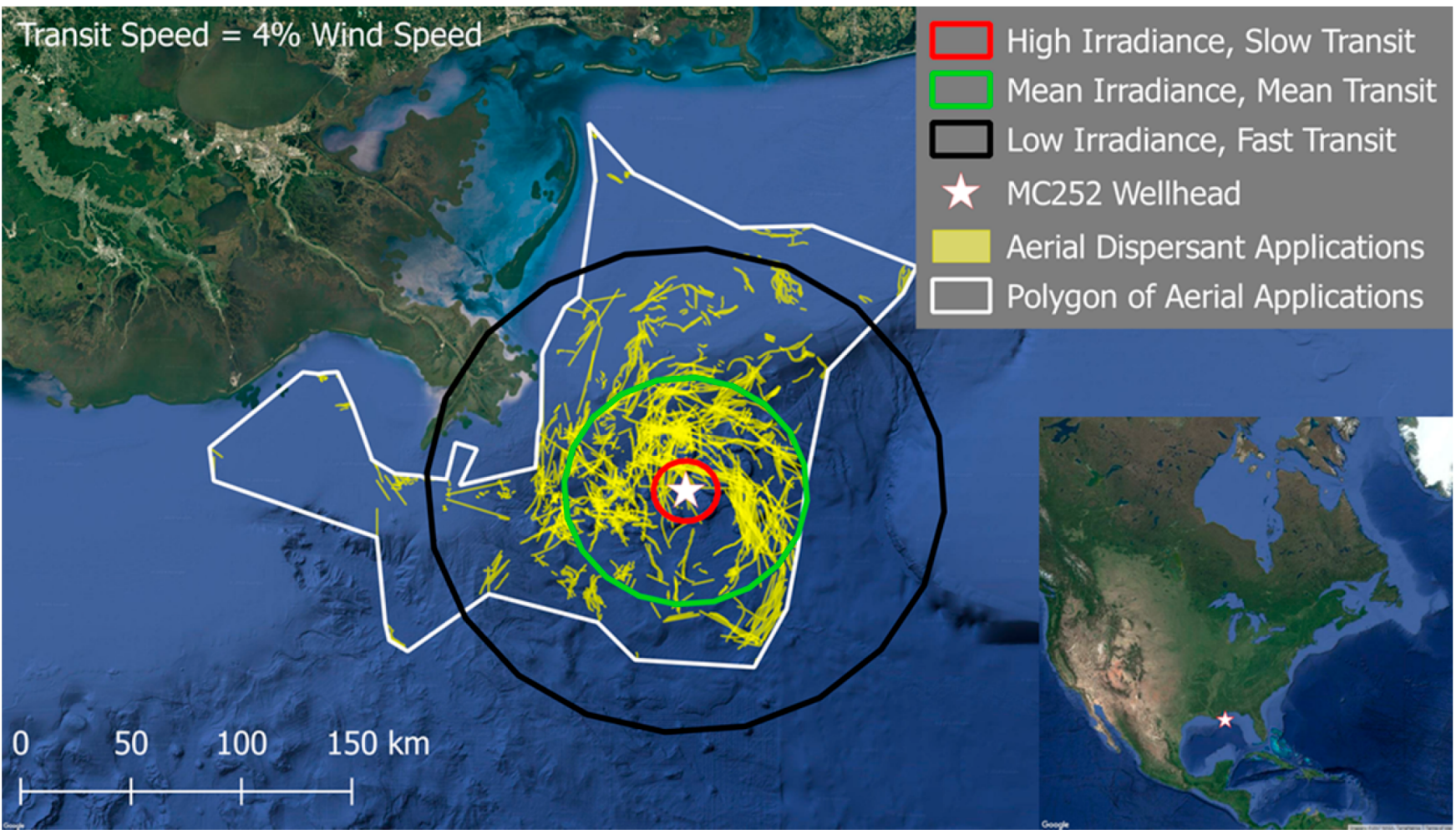

Figure 3. Assessing the effect of photochemical oxidation on the effectiveness of aerial dispersants applied in response to the DWH oil spill. The location of the Macondo well in the Gulf of Mexico is indicated by the white star. The locations of the 412 flight paths for aerial dispersant applications are colored yellow and are outlined by the polygon in white. Each concentric circle represents the distance DWH surface oil traveled before photo-oxidation decreased dispersant effectiveness to $<45 \%$, assuming high irradiance and slow transit speed (red inner circle), mean irradiance and transit speed (green intermediate circle), and low irradiance and fast transit speed (black outer circle). Irradiance levels were calculated by comparing the rate of simulated light absorption by oil in the laboratory vs natural sunlight on the Gulf of Mexico (Figure 1 and Figure S2). Transit speed was calculated as $4 \%$ of the average \pm SD of wind speed (Table S3). Transit speed was also calculated as 3 and $5 \%$ of wind speed (Table S3 and Figure S4), which did not change the conclusion from this analysis: a substantial fraction of aerial applications targeted oil that had low effectiveness (i.e., $<45 \%$ ), and this low effectiveness was principally driven by photochemical changes to the surface oil chemical properties.

of compounds that are insoluble in hexane and not amenable to gas chromatographic analysis, ${ }^{11,15,19,20}$ a chemical characteristic that has previously been reported to be a principle predictor of DE. ${ }^{7}$ Lastly, organic solvent is added to dispersant mixtures to facilitate the transfer of surfactants to the oil-water interface, a critical step in reducing oil-water interfacial tension and facilitating small droplet formation. ${ }^{2}$ However, photo-oxidized oil residues, containing at least 1 order of magnitude more oxygen than unaltered crude oil, ${ }^{11,15}$ are only partially soluble in the solvent system used in COREXIT EC9500A [i.e., $30 \pm 2 \%$ solubility in low-odor, low-aromatic food grade kerosene (Figure S3)]. This suppressed solubility indicates that the observed decrease in DE is likely driven by the incompatibility of the solvent system used in COREXIT EC9500A with photochemically transformed oil.

Constraining the Impact of Photo-Oxidation on Aerial Dispersant Effectiveness for the Deepwater Horizon Oil Spill. By combining estimates for (i) the time scale on which photo-oxidation decreased DE (Figure 1, Figure S2, and Appendix S1), (ii) transit speeds of oil floating on the Gulf of Mexico (Table S3 and Appendix S2), and (iii) the location of the 412 aerial chemical dispersant applications to DWH surface oil (yellow lines in Figure 3), ${ }^{21}$ we provide insights into the effectiveness of aerial dispersants applied in response to the DWH oil spill (Figure 3). The concentric circles in Figure 3 represent the minimum, mean, and maximum estimated distance that DWH surface oil traveled before photo-oxidation decreased $\mathrm{DE}$ to $<45 \%$ [i.e., $2-4$ days of transit time (Figure 1, Figure S2, and Table S3)]; the $\geq 45 \%$ threshold is a current listing requirement that dispersant manufacturers must achieve to be placed on the U.S. Environmental Protection Agency's National Contingency Plan Product Schedule so that it may be authorized for use in an oil spill. At average irradiance levels and oil transit speeds (i.e., $4 \%$ of average wind speed, $16 \mathrm{~km} \mathrm{day}^{-1}$ ), a substantial fraction of the 412 aerial applications targeted oil that had DE values of $<45 \%$, as evidenced by the hundreds of yellow lines that fall outside of the intermediate green concentric circle (Figure 3). At low irradiance levels and a fast oil transit speed $\left(25 \mathrm{~km} \mathrm{day}^{-1}\right)$, dozens of aerial dispersant applications likely did not achieve a DE of $45 \%$. Even assuming oil transit is $5 \%$ of wind speed, many applications would not meet the DE threshold of $45 \%$ (Figure S4). Furthermore, because winds (as well as surface currents) are not unidirectional, as assumed in this analysis, the oil transit speeds used in Figure 3 are likely overestimated. For example, transit times from the DWH well to Alabama, Mississippi, and Florida coasts were previously reported to range from 14 to 28 days, ${ }^{22}$ which correspond to oil transit speeds of $6-11 \mathrm{~km} \mathrm{day}^{-1}$. Assuming transit speeds of $6-11 \mathrm{~km}^{-1}{ }^{-1}$ and average irradiance levels, the distance that oil traveled before photo-oxidation decreased $\mathrm{DE}$ to $<45 \%$ lies between the red and green concentric circles in Figure 3, indicating that the majority of aerial applications targeted DWH surface oil with low DE. This analysis demonstrates that incorporating two commonly monitored 
environmentally variables into oil spill models, i.e., irradiance and wind speed, will refine predictions for the effectiveness of aerial dispersants applied in response to future spills in marine ecosystems.

Oil Spill Response Implications. This study challenges the paradigm that photochemical weathering has a negligible impact on the effectiveness of aerial dispersants applied in response to oil spills. In contrast to the current paradigm, our results indicate that the effects of photochemical weathering on the physical and chemical properties and dispersant effectiveness of Macondo well oil greatly outweigh the effects of evaporative weathering, a process regularly factored into oil spill response guidance. ${ }^{1,2,8-10}$ Pending future work to determine if these findings hold true for oil sources across a wide range of physical and chemical properties, models for predicting dispersant effectiveness, as well as standardized protocols for quantifying dispersant effectiveness, should consider the impact of photochemical oxidation and be modified accordingly. On the basis of our findings that photochemical weathering was likely a primary control of the dispersant effectiveness of Macondo well oil floating on the Gulf of Mexico, we propose that future oil spill guidance documents consider the impact of photochemical weathering on the "window of opportunity" to apply chemical dispersants in response to oil spills. The knowledge gained from this study enhances our capacity to respond effectively to future oil spills in sunlit waters, thereby minimizing risks to humans and ecosystems.

\section{ASSOCIATED CONTENT}

\section{S Supporting Information}

The Supporting Information is available free of charge on the ACS Publications website at DOI: 10.1021/acs.estlett.8b00084.

Appendices describing photochemical calculations; kinetic and geospatial modeling; tables showing physical properties of light-exposed oil; comparison of evaporation and photochemical oxidation impacts on physical properties; transit speed calculations; and figures showing the transmission of light through a glass pane, comparison of natural and simulated light absorption, kinetic and geospatial modeling at varying transit speeds, and the solubility of photo-oxidized oil (PDF)

\section{AUTHOR INFORMATION}

\section{Corresponding Author}

*Department of Marine Chemistry and Geochemistry, Woods Hole Oceanographic Institution, Woods Hole, MA 02543. Telephone: 508-289-3452. E-mail: cward@whoi.edu.

ORCID

Collin P. Ward: 0000-0003-2979-0280

Notes

The authors declare no competing financial interest.

\section{ACKNOWLEDGMENTS}

The authors thank Robert Holmes [Woods Hole Research Center (WHRC), Falmouth, MA], Jonathon Sanderman (WHRC), and Ben Van Mooy [Woods Hole Oceanographic Institution (WHOI)] for access to analytical equipment and John Farrington (WHOI), Philip Gschwend (Massachusetts Institute of Technology, Cambridge, MA), and Charles Sharples (University of Mary Washington, Fredericksburg, VA) for helpful discussions about our findings. Special thanks to Monica Noon for assistance with the GIS analysis. Thanks to three anonymous reviewers who substantially improved the quality of the manuscript. The irradiance data were provided by the U.S. Department of Agriculture UVB Monitoring Climatological and Research Network. This work was supported, in part, by National Science Foundation Grant OCE-1333148, Gulf of Mexico Research Initiative Grants 015, SA 16-30, the DEEP-C consortium, and the Clark Family Foundation, Inc. EPA funding was provided to R.N.C. from the Oil Spill Liability Trust Fund. All data are publicly available on the Gulf of Mexico Research Initiative Information and Data Cooperative (GRIIDC; doi:10.7266/N7D50KHM).

\section{REFERENCES}

(1) National Research Council. Using oil spill dispersants on the sea; National Academies Press: Washington, DC, 1989.

(2) National Research Council of the National Academies. Understanding oil spill dispersants: efficacy and effects; National Academies Press: Washington, DC, 2005.

(3) Prince, R. C.; Butler, J. D.; Redman, A. D. The rate of crude oil biodegradation in the sea. Environ. Sci. Technol. 2017, 51, 1278-1284.

(4) The Future of Dispersant Use in Oil Spill Response Initiative; Coastal Response Research Center and The National Oceanic Atmospheric Administration: Durham, NH, 2012; pp 1-252. https://crrc.unh.edu/sites/crrc.unh.edu/files/media/docs/ Workshops/dispersant_future_11/Dispersant_Initiative FINALREPORT.pdf (accessed January 1, 2017).

(5) Oil Budget Calculator Deepwater Horizon; Federal Interagency Solutions Group, Oil Budget Calculator Science and Engineering Team, 2010; pp 1-217. https://www.restorethegulf.gov/sites/default/ files/documents/pdf/OilBudgetCalc_Full_HQ-Print_111110.pdf (accessed January 1, 2017).

(6) Holder, E. L.; Conmy, R. N.; Venosa, A. D. Comparative laboratory-scale testing of dispersant effectiveness of 23 crude oils using four different testing protocols. J. Environ. Prot. 2015, 6, 628639.

(7) Fingas, M.; Wang, Z.; Fieldhouse, B.; Smith, P. The correlation of chemical characteristics of an oil to dispersant effectiveness. In Proceedings of the Twenty-Sixth Arctic and Marine Oilspill Program (AMOP) Technical Seminar; Environment Canada: Victoria, BC, 2003; Vol. 2, pp 679-730.

(8) Characteristics of Response Strategies: A Guide for Spill Response Planning in Marine Environments. National Oceanic and Atmospheric Administration, 2013; pp 1-76. https://response. restoration.noaa.gov/sites/default/files/Characteristics_Response_ Strategies.pdf (accessed January 1, 2017).

(9) Fingas, M. The Basics of Oil Spill Cleanup, 3rd ed.; CRC Press: Boca Raton, FL, 2013.

(10) Oil spill response field manual, 6th ed.; ExxonMobil Research and Engineering Co., 2014; pp 1-368. http://cdn.exxonmobil.com/ $\sim /$ media/global/files/energy-and-environment/oil-spill-response-fieldmanual_2014_e.pdf (accessed January 1, 2017).

(11) Ward, C. P.; Sharpless, C. P.; Valentine, D. L.; French-McCay, D.; Aeppli, C.; White, H. K.; Rodgers, R. P.; Gosselin, K. M.; Nelson, R. K.; Reddy, C. M. Partial photochemical oxidation was a dominant fate of Deepwater Horizon surface oil. Environ. Sci. Technol. 2018, 52, $1797-1805$.

(12) Reddy, C. M.; Arey, J. S.; Seewald, J. S.; Sylva, S. P.; Lemkau, K L.; Nelson, R. K.; Carmichael, C. A.; McIntyre, C. P.; Fenwick, J.; Ventura, G. T.; et al. Composition and fate of gas and oil released to the water column during the Deepwater Horizon oil spill. Proc. Natl. Acad. Sci. U. S. A. 2012, 109, 20229-20234.

(13) Hollebone, B. Oil physical properties: measurement and correlation. In Oil Spill Science and Technology; Fingas, M., Ed.; Elsevier: Cambridge, MA, 2017; pp 185-207.

(14) White, H. K.; Wang, C. H.; Williams, P. L.; Findley, D. M.; Thurston, A. M.; Simister, R. L.; Aeppli, C.; Nelson, R. K.; Reddy, C. 
M. Long-term weathering and continued oxidation of oil residues from the Deepwater Horizon spill. Mar. Pollut. Bull. 2016, 113, 380-386.

(15) Aeppli, C.; Carmichael, C. A.; Nelson, R. K.; Lemkau, K. L.; Graham, W. M.; Redmond, M. C.; Valentine, D. L.; Reddy, C. M. Oil weathering after the Deepwater Horizon disaster led to the formation of oxygenated residues. Environ. Sci. Technol. 2012, 46, 8799-8807.

(16) Sorial, G. A. Laboratory testing to determine dispersion predictability of the baffled flask test (BFT) and swirling flask test (SFT); Bureau of Safety and Environmental Enforcement (BSEE), U.S. Department of the Interior: Washington, DC, 2006.

(17) Sühring, R.; Smith, A.; Emerson, H.; Doran, D.; Mellor, P.; Kirby, M. F.; Christie, B. Qualification of oil-spill treatment products Adopting the Baffled Flask Test for testing of dispersant efficacy in the UK. Mar. Pollut. Bull. 2017, S0025-326X(17)30884-6.

(18) Comparison of large-scale (Ohmsett) and small-scale dispersant effectiveness test results; prepared by SL Ross Environmental Research for the U.S. Department of the Interior, Bureau of Ocean Energy Management, Regulation and Enforcement, Herndon, VA, 2011.

(19) Garrett, R. M.; Pickering, I. J.; Haith, C. E.; Prince, R. C. Photooxidation of crude oils. Environ. Sci. Technol. 1998, 32, 37193723.

(20) Radović, J. R.; Aeppli, C.; Nelson, R. K.; Jimenez, N.; Reddy, C. M.; Bayona, J. M.; Albaigés, J. Assessment of photochemical processes in marine oil spill fingerprinting. Mar. Pollut. Bull. 2014, 79, 268-277.

(21) Houma ICP Aerial Dispersant Group. After action report deepwater horizon MC252 aerial dispersant response; 2010, pp 1-80. https:// erma.noaa.gov/layerfiles / 28929 / files / MASTER\%20FINAL\%20After\%20Action\%20Report\%20Deepwa ter\% 20 Horizon $\% 20$ MC252\%20Aerial\%20 Dispersant $\% 20$ Res ponse\%20\%202011-02-09a1.pdf (accessed January 1, 2017).

(22) French-McCay, D.; Horn, M.; Li, Z.; Crowley, D.; Spaulding, M.; Mendelsohn, D.; Jayko, K.; Kim, Y.; Isaji, T.; Fonetanult, J.; et al. Simulation modeling of ocean circulation and oil spills in the Gulf of Mexico: Appendix VI data collection, analysis and model validation; Prepared by RPS ASA for the U.S. Department of the Interior, Bureau of Ocean Energy Management, Gulf of Mexico OCS Region, New Orleans, LA, 2017. 